\title{
Wnt3a Expression Is Associated with Epithelial- Mesenchymal Transition and Impacts Prognosis of Lung Adenocarcinoma Patients
}

\author{
Jinming $\mathrm{Xu}$, Wang Lv, Yeji Hu, Luming Wang, Yiqing Wang, Jinlin Cao, Jian $\mathrm{Hu}^{凶}$ \\ Department of Thoracic Surgery, The first Affiliated Hospital, Zhejiang University School of Medicine, Hangzhou 310003, China \\ $\triangle$ Corresponding author: Prof. Jian $\mathrm{Hu}, \mathrm{MD}, \mathrm{PhD}$. Department of Thoracic Surgery, The first Affiliated Hospital, Zhejiang University School of Medicine, \\ Hangzhou 310003, China. E-mail: dr_hujian@zju.edu.cn and hujian_med@163.com. Tel.: +86-0571-87236847 Fax: +86-0571-86995818 \\ (C) Ivyspring International Publisher. This is an open access article distributed under the terms of the Creative Commons Attribution (CC BY-NC) license \\ (https://creativecommons.org/licenses/by-nc/4.0/). See http://ivyspring.com/terms for full terms and conditions.
}

Received: 2016.11.30; Accepted: 2017.05.08; Published: 2017.08.02

\begin{abstract}
Background: Epithelial-mesenchymal transition (EMT) plays an important role in the invasion and migration during cancer metastasis. Wnt3a is one of the ligands in canonical Wnt/ 3 -catenin signaling pathway, which contributes to the carcinogenesis and progression of lung cancer cell lines. The aim of this study was to evaluate the association between Wnt3a and EMT-related proteins (E-cadherin and N-cadherin), and to further investigate its impact on prognosis of lung adenocarcinoma patients.

Methods: A total of 147 lung adenocarcinoma patients were included and their clinicopathological characteristics were collected in this retrospective study. The expression levels of Wnt3a, E-cadherin and $\mathrm{N}$-cadherin in post-surgery cancerous and adjacent normal tissues were assessed by immunohistochemistry. The association between Wnt3a and EMT-related proteins and their prognostic values were systematically evaluated. HCC827 and PC9 cell lines were treated with Wnt3a to detect the expression of EMT-related and $\mathrm{Wnt} / \beta$-catenin signaling-associated proteins, as well as the in vitro migration and invasion abilities.

Results: High Wnt3a expression level was significantly associated with low E-cadherin $(P<0.001)$ and high $\mathrm{N}$-cadherin $(P<0.001)$ expression levels in lung adenocarcinoma tissues. Besides, high Wnt3a level predicted poorer lung adenocarcinoma survival by univariate Cox analysis $(P=0.001)$, while the multivariate result was not significant $(P=0.355)$. Subgroup analysis suggested that the prognostic value of $W n t 3 a$ expression level was significant in stage T1-T2 (log rank $P=0.003)$ and stage N0 (log rank $P=0.031$ ) patients. The multivariate Cox analysis suggested $\mathrm{N}$-cadherin was an independent prognostic factor for lung adenocarcinoma patients $(P=0.012)$. After including these markers into a nomogram, the Harrell's $C$-index of the nomogram was 0.755 . The decision-curve analysis of our nomogram performed net benefit at the threshold probability from $21.6 \%$ to $82.0 \%$, and the current model had a better prognostic value than TNM-classification with a lower Akaike information criterion (AIC) value of 166.54. In vitro experiments suggested that $\mathrm{Wnt} 3$ a could regulate EMT-related proteins and promotes in vitro invasion and migration abilities.

Conclusions: Wnt3a could regulate EMT-related proteins and promote the migration and invasion process of lung adenocarcinoma. Although its value as an independent prognostic factor was limited, the combined model suggested good prognostic performance for lung adenocarcinoma patients.
\end{abstract}

Key words: Wnt3a expression, lung adenocarcinoma

\section{Introduction}

Lung cancer is the leading cause of cancer-related deaths in China and the world [1, 2], and non-small cell lung cancer (NSCLC) is the most common pathological type of all lung cancer cases [3]. Although surgical technique, chemotherapy and targeted therapy were significantly advanced, outcomes of NSCLC patients in different stages varied and the average five-year survival rate after diagnosis of primary NSCLC was only $18 \%$ [4]. Metastasis plays an important role in the progression of disease [5] and accounts for $90 \%$ of deaths from carcinomas [6]. Thus, it's necessary to investigate the underlying 
mechanisms of lung cancer metastasis to develop novel potential therapeutic targets.

Epithelial-mesenchymal transition (EMT) has been considered as a conceptual paradigm for the invasion and dissemination steps during cancer metastasis [7], in which cells lose epithelial features (decreased E-cadherin level) and gain mesenchymal properties (increased N-cadherin level) [8]. Besides, EMT could contribute to chemoresistance properties of tumor cells $[9,10]$ and targeting EMT pathways could be a promising therapeutic strategy. It has been reported that $\mathrm{Wnt} / \beta$-catenin signaling pathway plays an important role in EMT regulation [11, 12], and Wnt3a is one of the ligands in canonical Wnt/ $\beta$-catenin signaling pathway, which is well established in the progression of lung adenocarcinoma cell lines [13-15]. However, the clinicopathological significance of Wnt3a for NSCLC has not been comprehensively evaluated. In this study, we conducted a retrospective study and in vitro experiments to evaluate the association between Wnt3a expression and EMT-related proteins (E-cadherin and N-cadherin), and to further investigate their impacts on the prognosis of lung adenocarcinoma patients.

\section{Materials and Methods}

\section{Lung adenocarcinoma tissues}

A total of 147 stage I-III lung adenocarcinoma patients were included between July 2004 and June 2009 in the First Affiliated Hospital of Zhejiang University (Hangzhou, China). Primary lung adenocarcinoma cancerous tissues (C) and corresponding adjacent normal tissues $(\mathrm{N})$ were collected after surgery and their clinicopathological characteristics were collected from medical records and pathological reports. All tissue specimens were fixed with $10 \%$ neutral formalin solution and were paraffin-embedded after routine dehydration. The pathological features and clinical stage of primary tumors were defined on the basis of criteria of the American Joint Commission on Cancer/Union for International Cancer Control (AJCC/UICC $7^{\text {th }}$ version) tumor-node-metastasis (TNM) staging manuals. No patients received adjuvant treatment including radiotherapy or chemotherapy prior to surgery and diagnosis, and all patients were enrolled with written informed consent under institutional review board-approved protocols of the First Affiliated Hospital of Zhejiang University. All experiments in this study were performed in accordance with the principles of Declaration of Helsinki.

Immunohistochemistry was conducted to evaluate expression levels of Wnt3a, E-cadherin and $\mathrm{N}$-cadherin in formalin-fixed specimens. A scoring criterion was applied to the immunohistochemistry result determination. The intensity of marker staining was scored as 0 (no signal), 1 (weak), 2 (moderate), and 3 (strong). The percentage of positive cells was also scored as $0(<10 \%), 1(11 \%-30 \%), 2$ (31\%-50\%) and $3(>50 \%)$. The markers were classified as high expression if the total score was equal to or more than three points; otherwise it was classified as low expression. The immunohistochemistry results were reviewed independently by two authors. Discrepancies were revaluated until a consensus reached. The last follow up time was August 2014 and the mean survival time was 3.86 years.

\section{Cell lines and cell culture}

Human lung adenocarcinoma cells HCC827 and PC9 were purchased from the American type culture collection (ATCC, The Global Bioresource Center, Manassas, VA, USA), and they were cultured in RPMI 1640 medium (BD Biosciences, San Jose, CA, USA) containing $10 \%$ fetal bovine serum (FBS) and maintained in $37^{\circ} \mathrm{C}, 5 \% \mathrm{CO}_{2}$, saturated humidity cell culture. For Wnt3a administration in vitro, Wnt3a (100ng/ml) was obtained from R\&D systems. Cells were harvested 72 hours post-treatment and all the cell lysates were collected and measured by Western blot.

\section{Western blot}

Cells were cultured for 72 hours after treatment with Wnt3a $(100 \mathrm{ng} / \mathrm{ml})$. The total protein of human lung adenocarcinoma HCC827 and PC9 cells was extracted by DC method. The $\beta$-catenin protein in cytoplasm and nucleus was extracted by the NE-PER ${ }^{\mathrm{TM}}$ Nuclear and Cytoplasmic Extraction Reagents (Rockford, IL, USA). The protein samples were analyzed by PAGE-SDS electrophoresis and wet-transferred to PVDF membrane. Membranes were blocked in 3\% BSA for 1 hour, and incubated with primary antibody for 2 hours at room temperature. After TBST washing, membrane was incubated for 1 hour with HRP-labeled secondary antibody, and washed 3 times with TBST. The expression of protein was analyzed by adding ECL to the gel imaging system. GAPDH and Lamin A/C were used as the internal reference proteins in the cytoplasm and the nucleus, respectively. All the antibodies used for Western blot were purchased from the CST Company (Danvers, MA, USA).

\section{Migration and invasion assays}

For migration assays, HCC827 and PC9 cells treated with or without Wnt3a were placed in the upper Transwell chamber $\left(2 \times 10^{4}\right.$ cells/well) (Corning 
Company, Cambridge, MA, USA). RPMI 1640 medium containing $10 \%$ FBS was placed on the bottom chamber. The lower surface of $6.5-\mathrm{mm}$ polycarbonate filters was coated by immersion in $0.1 \%$ gelatin. Cell cultures were incubated at $37^{\circ} \mathrm{C}$ for 24 hours and the non-invading cells were removed. The invading cells were fixed with $4 \%$ paraformaldehyde for 15 minutes and stained by $2 \%$ crystal violet for 30 minutes. For invasion assays, filters (8- $\mu \mathrm{m}$ pore) coated with Matrigel basement membrane matrix (BD Biosciences, San Jose, CA) were used, and the other experiment process was the same as described for the cell migration assays.

\section{Statistical analysis}

The overall survival was selected as the outcome of interests for this study. Pearson Chi-square test was adopted to assess the association between Wnt3a and EMT-related proteins (E-cadherin and N-cadherin). Univariate and multivariate cox regression analyses and Kaplan-Meier curves were conducted and then a nomogram model was constructed to further evaluate the prognostic value of these markers. The discrimination efficacy of the nomogram was assessed by concordance index (C-index), which ranged from 0.5 (no discrimination) to 1 (high discrimination). Besides, the calibration efficacy was assessed by comparing the predicted survival with the observed survival, and bootstrap technique with 1000 repetitions was used for internal validation [16, 17]. The detailed code for building a nomogram was shown in the Supplementary File. The optimal cut-off values were determined using $X$-tile software (http://www.tissuearray.org/rimmlab) and by the minimal $P$-value approach [18]. In addition, a decision curve analysis was also adopted to assess the net benefit of nomogram-assisted decisions at different threshold probabilities [19]. The detailed information and documentation for decision-curve analysis is publicly available at www.decisioncurveanalysis.org. Akaike information criterion (AIC) analysis was then applied to further evaluate the predictive efficacy for nomogram and traditional TNM models, and smaller AIC values represent more accurate prognostic stratification [20]. In the statistical analysis process, subgroups that had a relative small sample size were combined together.

All analyses were conducted using the SPSS 19.0 software (IBM SPSS Inc. United States), Stata software (version 12.0; StatCorp, College Station, TX, USA), and R software version 3.2.2 (The R Foundation for Statistical Computing) with the rms and Hmisc statistical packages. Statistical significance was set at $P<0.05$ (All $P$ values presented were 2-sided).

\section{Results}

\section{Basic characteristics of subjects}

The basic characteristics of included subjects were summarized in Table 1. AJCC stage IV and stage N3 patients were excluded, and a total of 147 patients were included. The mean age was 61 years. Due to the small sample size of stage T4 patients $(n=4)$, we combined them with the stage T3 group. The mean survival time was 3.86 years and the overall survival rate was $46.3 \%$. According to the immunohistochemistry results, cancerous tissues and adjacent normal tissues were categorized as high and low expression of Wnt3a, E-cadherin and N-cadherin (Figure 1A). Among the 147 cancerous tissues, 38 $(25.9 \%)$ showed high Wnt3a expression, while only 14 (9.5\%) adjacent normal tissues showed high Wnt3a expression, which indicated Wnt3a may be involved in the carcinogenesis of lung adenocarcinoma.

\section{The prognostic value of Wnt3a in lung adenocarcinoma patients}

Cox regression analysis and Kaplan-Meier curve were adopted to evaluate the prognostic value of Wnt3a and EMT-related proteins in lung adenocarcinoma patients. Univarite analysis was first conducted, and then multivariate analysis was performed based on statically significant factors in the univarite analysis. According to the results in Table 1 and Figure 1B, high Wnt3a expression level in cancerous lung adenocarcinoma tissues predicted poorer survival, with a hazard ratio (HR) and 95\% confidence interval $(\mathrm{CI})$ of 2.214(1.386-3.536, $P=0.001$ ) and the Kaplan-Meier curve suggested a well discrimination and the log rank (Mantel-Cox) $P$ value was 0.001 . However, the multivariate result was not significant $(\mathrm{HR}=1.285$, 95\% CI: 0.756-2.184, $P=0.355)$. The Kaplan-Meier curves of E-cadherin and $\mathrm{N}$-cadherin were shown in Figure 1C and 1D, with log rank $P$ value of 0.002 and $<0.001$, respectively. Besides, the multivariate cox analysis suggested that high $\mathrm{N}$-cadherin level $(1.984,1.164-3.381, P=0.012)$, age $\geq 70$ years $(1.869,1.139-3.068, P=0.013)$, and lymph node metastasis (stage N1 2.522, 1.366-4.656, $P=0.003$ and stage N2 4.722, 2.521-8.847, $P<0.001$ ) were independent prognostic factors for lung adenocarcinoma patients.

Subgroup analyses stratified by $\mathrm{T}$ stage and $\mathrm{N}$ stage were also conducted. The prognostic value of Wnt3a in different stages was assessed by Kaplan-Meier curve and log rank test. High Wnt3a level predicted poorer prognosis in stage T1-T2 patients $(P=0.003$, Figure $2 \mathrm{~A})$ and stage N0 patients $(P=0.031$, Figure $2 C)$, which indicated that Wnt3a might be more sensitive in early stage lung 
adenocarcinoma. The subgroup analysis results of E-cadherin and $\mathrm{N}$-cadherin were shown in Supplementary Figure 1 and 2 and $\mathrm{N}$-cadherin had a relatively better prognostic value in stage T1-T2, T3-T4 and N1-N2 patients.

\section{The association between Wnt3a and EMT-related proteins}

The immunohistochemistry and Pearson Chi-square analysis results suggested that high Wnt3a expression level was significantly associated with low E-cadherin and high $\mathrm{N}$-cadherin expression levels in lung adenocarcinoma tissues, as shown in Figure 1 and Table 2. The $\chi^{2}$ and $P$-value were 16.321, $P<0.001$ and 25.060, $P<0.001$, respectively. The E-cadherin and the N-cadherin are the features of epithelial cells and mesenchymal cells, and the result indicated that Wnt3a could promote the EMT process and may play a critical role in the tumor progression.

The association between Wnt3a, EMT-related markers and clinical characteristics and lymph node metastasis in lung adenocarcinoma patients was also assessed by Pearson Chi-square test, as shown in
Supplementary Table 1. High cancerous tissue levels of N-cadherin was significantly associated with lymph node metastasis and the $\chi^{2}$ was 17.025 and the $P$-value was $<0.001$. However, there was no significant difference of lymph node metastasis between high and low Wnt3a group $(P=0.077)$.

\section{A nomogram based on Wnt3a and EMT-related markers to predict lung adenocarcinoma survival}

Although the multivariate cox regression didn't provide evidence that Wnt3a was an independent prognostic factor, the Kaplan-Meier curves for overall, stage T1-T2 and N0 patients indicated potential discrimination efficacy. In order to further evaluate its role in lung adenocarcinoma prognosis, we combined it with EMT-related proteins and clinopathological factors and constructed a nomogram model, as shown in Figure 3A. During the variable selection process, the number of variables was restricted and the number of events should be greater than 10 times the number of predictors [16], otherwise, the expected error of the Cox predicted model would be more than
A

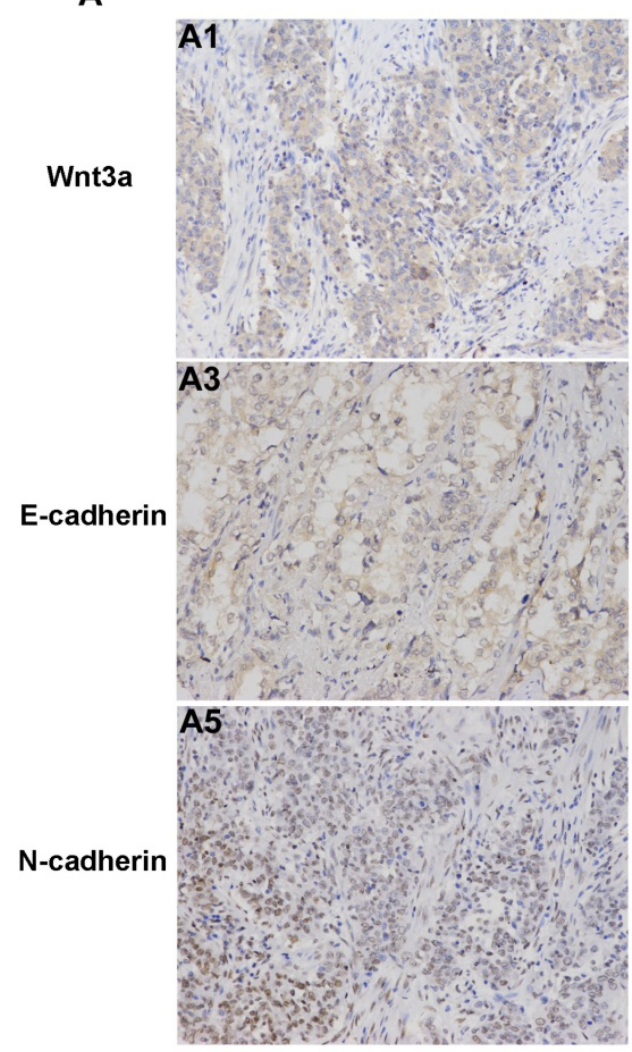

Sample I
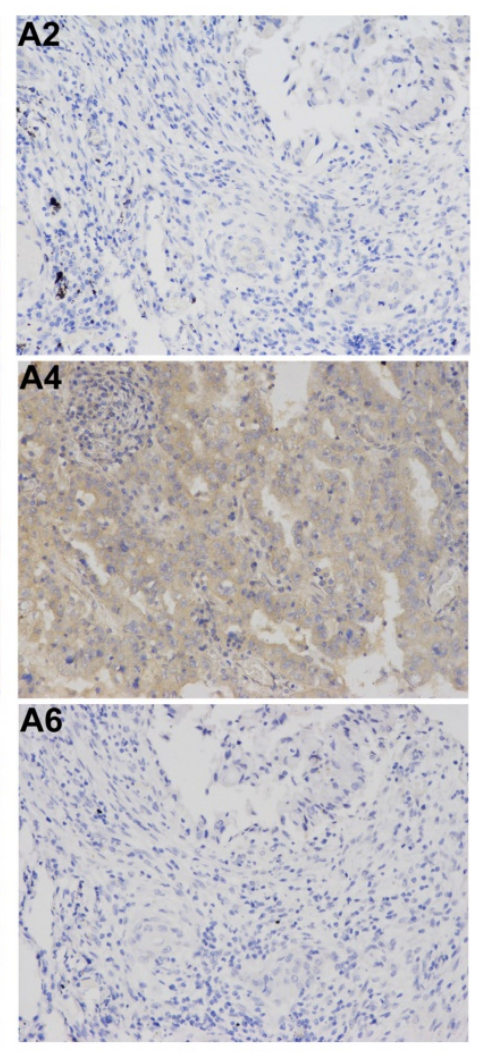

Sample II
B

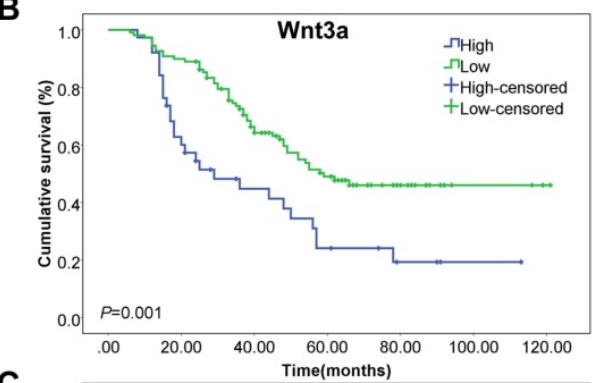

C

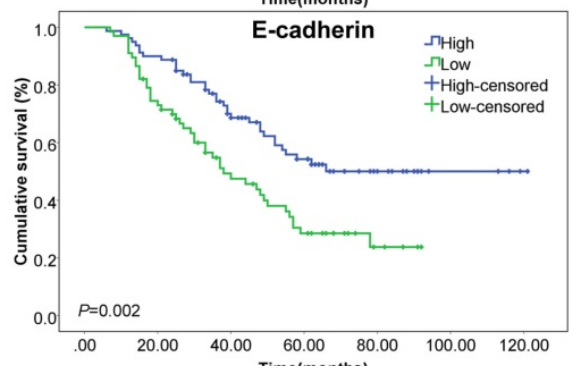

D

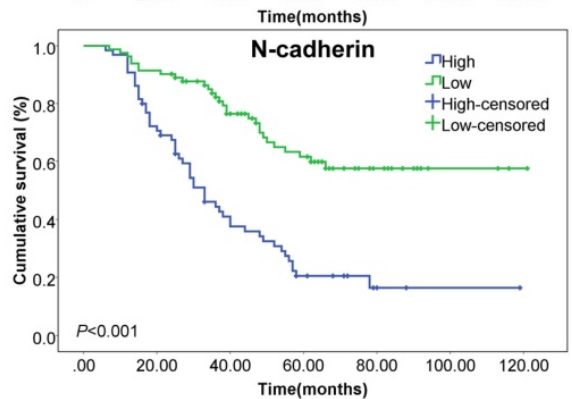

Figure 1. Immunohistochemistry results of Wnt3a and EMT features in human lung adenocarcinoma tissue samples and prognostic Kaplan-Meier curves of Wnt3a. (A) sample I was representative NSCLC sample with Wnt3a high expression (A1), E-cadherin low expression (A3) and N-cadherin high expression (A5); sample II was representative lung adenocarcinoma sample with Wnt3a low expression (A2), E-cadherin high expression (A4) and $\mathrm{N}$-cadherin low expression (A6) (200×magnification). (B) Kaplan-Meier curve for Wnt3a in lung adenocarcinoma patients, with Log Rank test $P$ value 0.001 . (C) Kaplan-Meier curve for E-cadherin in lung adenocarcinoma patients, with Log Rank test $P$ value 0.002 . (D) Kaplan-Meier curve for $N$-cadherin in lung adenocarcinoma patients, with Log Rank test $P$ value $<0.001$. 
$10 \%$. Finally, Wnt3a, E-cadherin, N-cadherin, age, $\mathrm{T}$ and $\mathrm{N}$ stage were selected as predictors for the 3-year survival of lung adenocarcinoma patients. The Harrell's C-index and 95\% CI of the nomogram was $0.755(0.697-0.813)$, which was better than the seventh edition of the AJCC-TNM classification (C index 0.710, 95\% CI: 0.648-0.772). The calibration efficacy performed a good fitness at the time of 3-year follow up (Figure 3B), indicating the predicted survival probabilities were consistent with the actual observations. X-tile analysis was conducted and the optimal cut-off scores were 1.54 to $12.50,12.50$ to 15.88 and 15.88 to 22.59 points (Figure 3C), and the 3-year survival rate were $73.08 \%, 58.06 \%$ and $23.68 \%$, respectively $(P<0.001)$. In addition, a decision curve analysis was applied to assess the clinical usefulness of the nomogram (Figure 3D). In our study, the current nomogram model showed net benefit if the patients' predicted 3-year survival probability ranged from $21.6 \%$ to $82.0 \%$, and more intensive treatments and follow-up might need recommendation. The current nomogram model performed better net benefit than the traditional AJCC-TNM classification (3-year probability ranged from $31.8 \%$ to $71.0 \%$ ). The AIC values for nomogram and traditional TNM models were 166.54 and 177.44 , respectively, which suggested the nomogram model was better to explain variation and had better predictive accuracy than TNM staging model.

\section{Wnt3a could induce EMT and promote in vitro migration and invasion abilities}

Wnt3a treatment $(100 \mathrm{ng} / \mathrm{ml}, 72$ hours) could activate the classic Wnt/ $\beta$-catenin signaling pathway by enhancing the expression of membrane receptor low-density-lipoprotein receptor-related protein (LRP6) and adaptor protein Dishevelled 2 (Dv12) in HCC827 and PC9 cell lines, as shown in Figure 4A. The expression of cytoplasmic $\beta$-catenin decreased and nuclear $\beta$-catenin increased after Wnt3a treatment, which suggested that $\beta$-catenin accumulated in the nucleus and further produced biological functions. Besides, Wnt3a treatment could induce mesenchymal phenotype and promote the EMT process, as shown in Figure 4B. Compared with the untreated HCC827 and PC9 cell lines, the expression of epithelial marker (E-cadherin) decreased and the expression of mesenchymal maker (Vimentin) increased after Wnt3a treatment. The expression of EMT-related transcript factors (Snail and Slug) was also up-regulated. Furthermore, Wnt3a treatment could promote in vitro migration and invasion abilities of HCC827 and PC9 cell lines, as shown in Figure 4C.

Table 1. Characteristics of subjects and Cox regression analyses of survival in lung adenocarcinoma patients.

\begin{tabular}{|c|c|c|c|c|c|c|c|c|}
\hline Factor & Category & Total & Event & Percent & Univariate HR & $P$-value & Multivariate HR & $P$-value \\
\hline \multirow[t]{2}{*}{ Wnt3a(C) } & low & 109 & 52 & $47.71 \%$ & 1 & & 1.000 & \\
\hline & high & 38 & 27 & $71.05 \%$ & $2.214(1.386-3.536)$ & 0.001 & $1.285(0.756-2.184)$ & 0.355 \\
\hline \multirow[t]{2}{*}{ Wnt3a(N) } & low & 133 & 71 & $53.38 \%$ & 1 & & & \\
\hline & high & 14 & 8 & $57.14 \%$ & $1.277(0.614-2.655)$ & 0.512 & & \\
\hline \multirow[t]{2}{*}{ E-cadherin $(C)$} & low & 67 & 44 & $65.67 \%$ & 1 & & 1.000 & \\
\hline & high & 80 & 35 & $43.75 \%$ & $0.487(0.318-0.776)$ & 0.002 & $0.741(0.449-1.224)$ & 0.242 \\
\hline \multirow[t]{2}{*}{ E-cadherin(N) } & low & 35 & 17 & $48.57 \%$ & 1 & & & \\
\hline & high & 112 & 62 & $55.36 \%$ & $1.138(0.665-1.947)$ & 0.638 & & \\
\hline \multirow[t]{2}{*}{ N-cadherin $(C)$} & low & 82 & 29 & $35.37 \%$ & 1 & & 1.000 & \\
\hline & high & 65 & 50 & $76.92 \%$ & $3.246(2.046-5.150)$ & $<0.001$ & $1.984(1.164-3.381)$ & 0.012 \\
\hline \multirow[t]{2}{*}{ N-cadherin(N) } & low & 127 & 67 & $52.76 \%$ & 1 & & & \\
\hline & high & 20 & 12 & $60.00 \%$ & $1.162(0.628-2.149)$ & 0.632 & & \\
\hline \multirow[t]{2}{*}{ Smoke } & $\mathrm{N}$ & 82 & 45 & $54.88 \%$ & 1 & & & \\
\hline & $\mathrm{Y}$ & 65 & 34 & $52.31 \%$ & $0.992(0.590-1.440)$ & 0.922 & & \\
\hline \multirow[t]{2}{*}{ Gender } & $\mathrm{F}$ & 68 & 36 & $52.94 \%$ & 1 & & & \\
\hline & $\mathrm{M}$ & 79 & 43 & $54.43 \%$ & $1.079(0.693-1.681)$ & 0.736 & & \\
\hline \multirow[t]{2}{*}{ Age $^{*}$} & $<70$ & 113 & 54 & $47.79 \%$ & 1 & & 1.000 & \\
\hline & $\geq 70$ & 34 & 25 & $73.53 \%$ & $2.067(1.283-3.332)$ & 0.003 & $1.869(1.139-3.068)$ & 0.013 \\
\hline \multirow{2}{*}{ Pathological differentiation } & poor & 46 & 25 & $54.35 \%$ & 1 & & & \\
\hline & moderate and well & 101 & 54 & $53.47 \%$ & $0.884(0.550-1.421)$ & 0.611 & & \\
\hline \multirow[t]{3}{*}{ T stage } & $\mathrm{T} 1$ & 32 & 10 & $31.25 \%$ & 1 & & 1.000 & \\
\hline & $\mathrm{T} 2$ & 83 & 47 & $56.63 \%$ & $2.120(1.070-4.201)$ & 0.031 & $1.487(0.708-3.125)$ & 0.295 \\
\hline & T3-4 & 32 & 22 & $68.75 \%$ & $2.939(1.387-6.227)$ & 0.005 & $0.970(0.406-2.320)$ & 0.945 \\
\hline \multirow[t]{3}{*}{ N stage } & N0 & 80 & 25 & $31.25 \%$ & 1 & & 1.000 & \\
\hline & N1 & 31 & 22 & $70.97 \%$ & $2.803(1.577-4.983)$ & $<0.001$ & $2.522(1.366-4.656)$ & 0.003 \\
\hline & $\mathrm{N} 2$ & 36 & 32 & $88.89 \%$ & $6.095(3.549-10.469)$ & $<0.001$ & $4.722(2.521-8.847)$ & $<0.001$ \\
\hline
\end{tabular}

Univariate and multivariate analyses were conducted based on Cox regression model.

C: Primary lung adenocarcinoma cancerous tissues

$\mathrm{N}$ : Corresponding adjacent normal tissues 
A
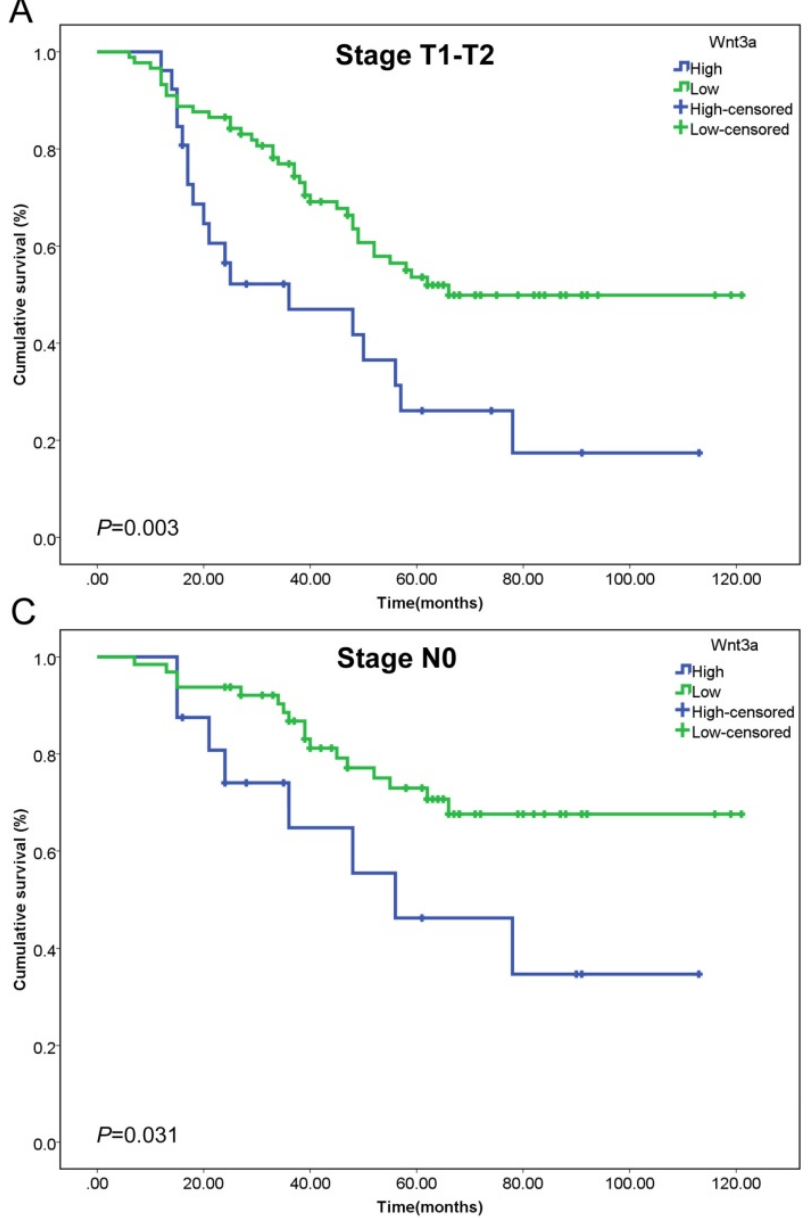

B

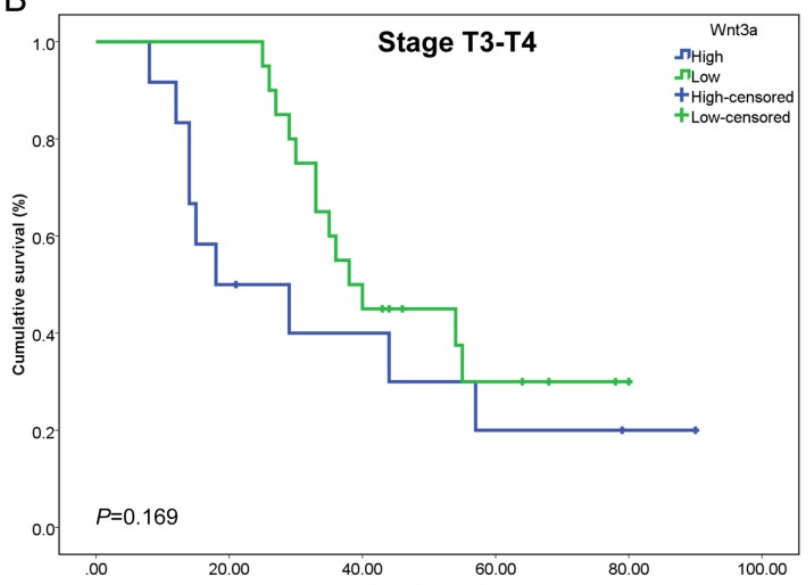

D

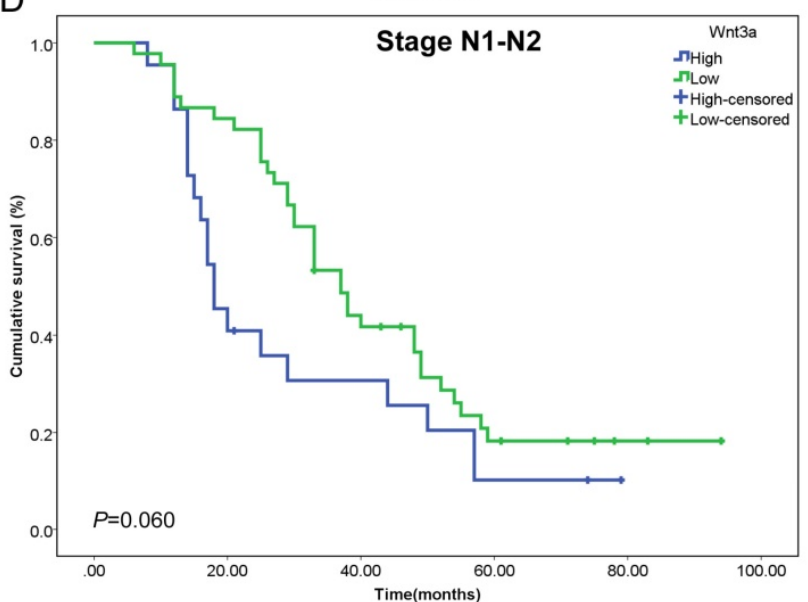

Figure 2. Kaplan-Meier curves of $W n t 3 a$ stratified by $T$ stage and $N$ stage. The prognostic value of Wnt3a was assessed in $(A)$ stage $T 1-T 2$ patients $(P=0.003)$, $(B)$ stage T3-T4 patients $(P=0.169),(C)$ stage N0 patients $(P=0.031)$, and $(D)$ stage N1-N2 patients $(P=0.060)$.

A

Points

Wnt3a

E cadherin

$\mathrm{N}$ cadherin

Age

T stage

$\mathrm{N}$ stage

Total Points

3-year survival

C

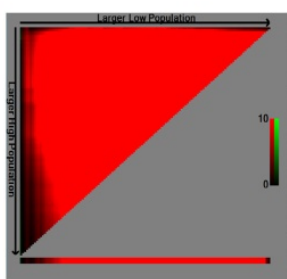

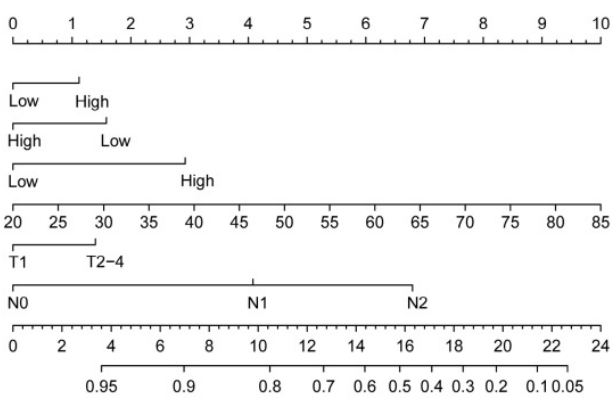

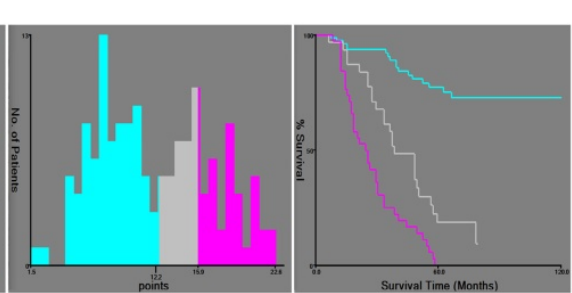

B

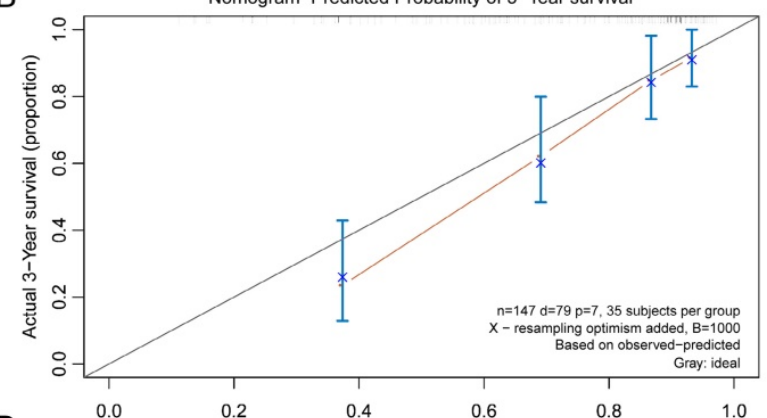

D

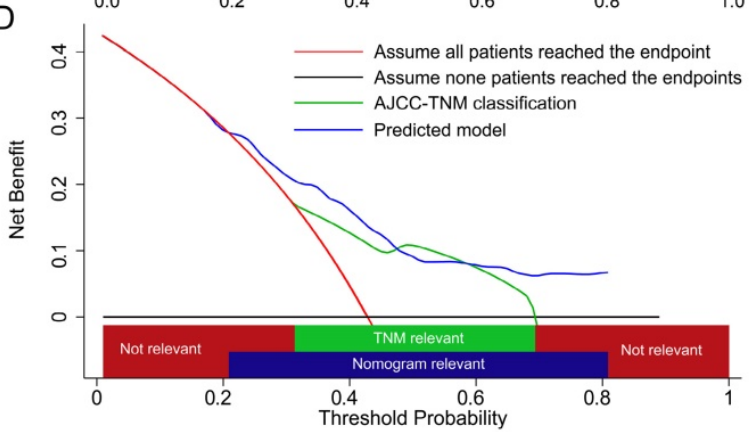

Figure 3. A nomogram predicted survival risk based on Wnt3a, EMT-related proteins and other factors in patients with lung adenocarcinoma. (A) The nomogram created by $\mathrm{R}$ language with a $\mathrm{C}$-index of 0.755 . (B) The calibration curves for predicting patient survival at 3-year. (C) $\mathrm{X}$-tile analysis of survival based on risk scores (cut off intervals: 1.54 to $12.50,12.50$ to 15.88 and 15.88 to 22.59 ). (D) Decision curve analysis of the nomogram. The net benefits (y axis) is plotted against the threshold probabilities of survival on the $x$ axis (3-year survival probability of nomogram model and TNM model ranged from $21.6 \%$ to $82.0 \%$, and from $31.8 \%$ to $71.0 \%$, respectively). 
A

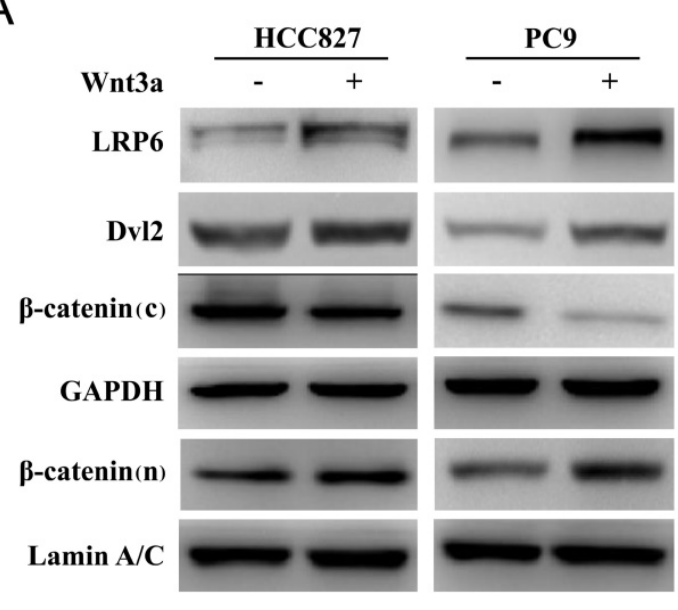

B

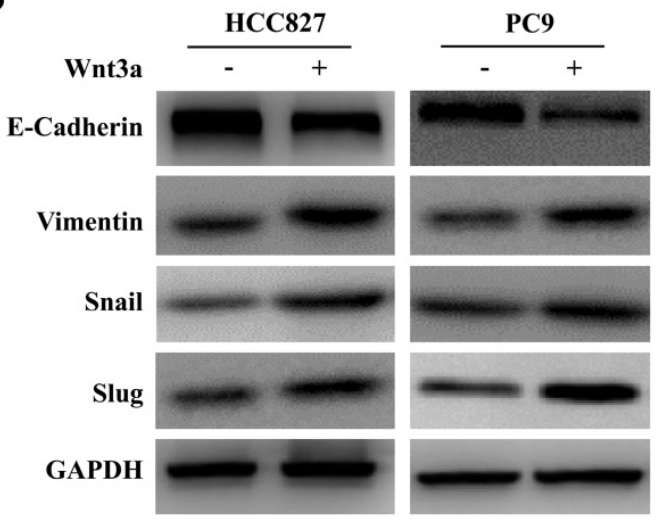

C
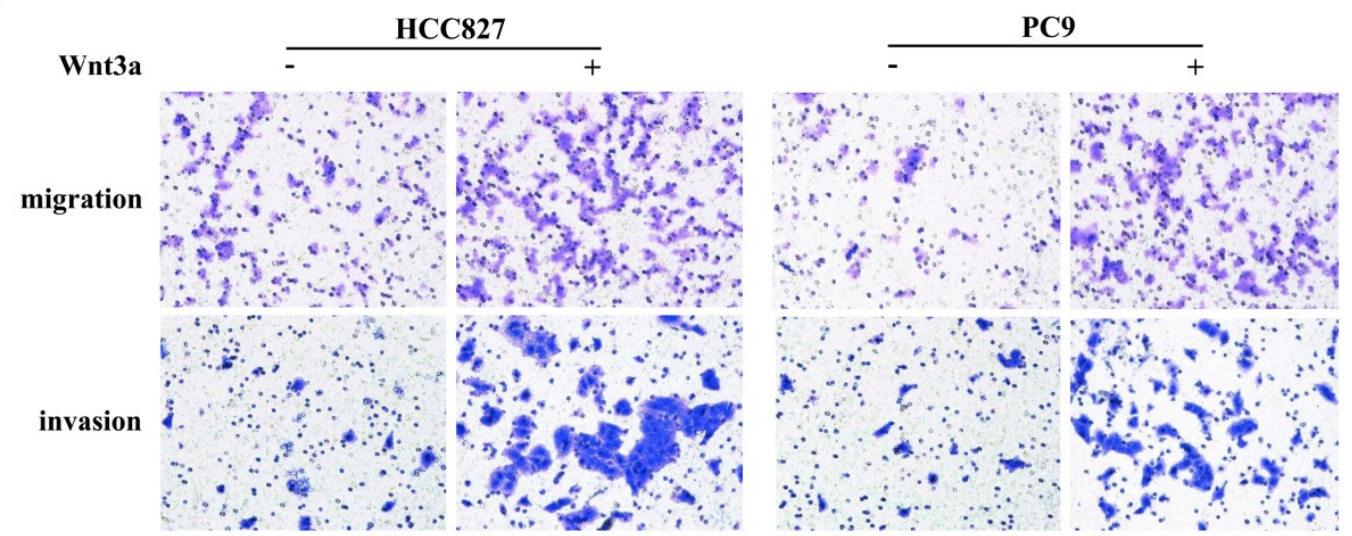

Figure 4. Wnt3a induced EMT and promoted in vitro invasion and migration abilities. (A) Wnt3a treatment (100ng/ml, 72 hours) could activate the classic Wnt/ $\beta$-catenin signaling pathway by enhancing the expression of membrane receptor LRP6 and adaptor protein Dvl2 in HCC827 and PC9 cell lines ( $\beta$-catenin (c): cytoplasm; $\beta$-catenin (n): nucleus). (B) Wnt3a treatment could induce mesenchymal phenotype and promote the EMT process. (C) Wnt3a treatment could promote in vitro migration and invasion abilities of $\mathrm{HCC} 827$ and PC9 cell lines.

Table 2. The association between Wnt3a and EMT-related proteins (E-cadherin and $\mathrm{N}$-cadherin) expression levels.

\begin{tabular}{lll}
\hline Factors & \multicolumn{2}{l}{ Wnt3a expression level } \\
\cline { 2 - 3 } & low $(\mathrm{n}=109)$ & high $(\mathrm{n}=38)$ \\
\hline E-cadherin expression & $70(64.22 \%)$ & $10(26.32 \%)$ \\
high (\%) & $39(35.78 \%)$ & $28(73.68 \%)$ \\
low (\%) & 16.321 & \\
$\chi^{2}$ & $<0.001$ & \\
$P$-value & & $30(78.95 \%)$ \\
N-cadherin expression & $35(32.11 \%)$ & $8(21.05 \%)$ \\
high (\%) & $74(67.89 \%)$ & \\
low (\%) & 25.060 & \\
$\chi^{2}$ & $<0.001$ & \\
$P$-value & & \\
\hline
\end{tabular}

$P$-value was tested by Pearson Chi-square analysis

\section{Discussion}

The results of the present study indicated that Wnt3a played an important role in the EMT process of lung adenocarcinoma. Although there is insufficient evidence to support Wnt3a as an independent prognostic marker, the nomogram model based on Wnt3a and EMT-related proteins performed good prognostic value over the AJCC-TNM classification and the nomogram calibration curve performed good fitness at 3-year follow up. The Kaplan-Meier curves of Wnt3a showed significant divergences, especially for stage T1-T2 and N0 patients, which suggested that Wnt3a may have a potential prognostic value for early stage lung adenocarcinoma patients.

Calibration and discrimination are two main aspects when validating the monogram and avoid overfitting [16, 17]. The C-index of our nomogram was 0.755 and the calibration curve for 3-year follow up showed consistence between the prediction probabilities and actual observations, which suggested that this model was well established and had the potential to be applied generally. Decision curve analysis was first reported by Vickers and Elkin et al. [19], and then applied and discussed by many studies $[17,21-23]$. If the probability of the disease or outcome is too high or too low, the decision is generally clear [24]. Thus, decision curve analysis provided further assessment of the clinical usefulness of a prediction model in a specific range of interest. In 
our analysis, the endpoint in decision curve analysis was set as 3-year survival, and the cut-off threshold probability ranged from $21.6 \%$ to $82.0 \%$. In other words, if a patient's 3-year survival probability predicted by our nomogram was in this range, nomogram-assisted decisions are relevant and the net benefit is better than the traditional TNM classification model. Besides, the AIC analysis also suggested the nomogram model had more accurate prognostic stratification.

The mechanisms of Wnt3a and EMT process have not been thoroughly understood. It has been reported the Wnt3 gene was significant associated with cell cycle regulators, oncogenes, and matrix degrading enzymes, which play important roles in tumor progression and metastatic potential in NSCLC $[11,15]$. As a ligand that activates the canonical Wnt/ $\beta$-catenin signaling pathway, secreted Wnt proteins can bind to the receptor complex consisting of the Frizzled and the LRP 5/6 receptors. The adaptor protein Dvl is subsequently phosphorylated, which promotes $\beta$-catenin accumulation and translocation into the nucleus. The $\beta$-catenin interacts with TCF/LEF transcription factors to activate transcription of Wnt targeting genes [25-28]. The western blot results suggested that Wnt3a treatment could enhance the expression of membrane receptor LRP6 and adaptor protein Dvl2 in HCC827 and PC9 cell lines, and $\beta$-catenin accumulated in the nucleus. The transcription factors Snail/Slug are known to repress E-cadherin and promote a mesenchymal phenotype [29, 30]. Our results also suggested that Wnt3a treatment could induce mesenchymal phenotype and promote the EMT process and further promote in vitro migration and invasion abilities.

The prognostic values of E-cadherin and $\mathrm{N}$-cadherin have been assessed by many studies [31-34], and our results indicated high N-cadherin could be considered as useful biomarkers for predicting the prognosis of lung adenocarcinoma, which was consistent with the previous study [34]. In addition, EMT was not only involved in the tumor metastasis, but also associated with the primary drug resistance of epidermal growth factor receptor-tyrosine kinase inhibitor (EGFR-TKI) and chemotherapy $[9,10,35,36]$, which was a bottleneck of targeted therapy in NSCLC. The current study provided evidence that Wnt3a played a pivotal role in the EMT process form both clinical and experimental points of view, and may help to identify novel potential therapeutic targets to improve the EGFR-TKI resistance. However, no significant association between Wnt3a expression level and lymph node metastasis was found. In fact, the role of EMT in tumor metastasis and the mechanisms were controversial. Fischer et al. [10] reported that the EMT transition was not required for metastasis but contributed to chemoresistance, and there existed great importance to develop EMT-targeting therapies [37]. Thus, more well-designed studies might be needed to further illustrate this issue.

Our study has several advantages. We provided evidence for the role of Wnt3a in the EMT process from both clinical and experimental perspectives and further revealed its prognostic value for early stage lung adenocarcinoma patients. Besides, we made comprehensive assessment of its prognostic value by univariate, multivariate cox analyses, nomogram model, decision curve analysis and AIC analysis. The combined model showed better predictive performance than the traditional TNM classification.

In conclusion, we found that Wnt3a could regulate EMT-related proteins and promote the migration and invasion process of lung adenocarcinoma. Although its prognostic value was limited as one individual marker, the combined model suggested good prognostic performance for lung adenocarcinoma patients.

\section{Abbreviations}

EMT: epithelial-mesenchymal transition; AIC: Akaike information criterion; NSCLC: non-small cell lung cancer; AJCC/UICC: the American Joint Commission on Cancer/Union for International Cancer Control; TNM: tumor-node-metastasis; FBS: fetal bovine serum; C-index: concordance index; HR: hazard ratio; $\mathrm{CI}$ : confidence interval; LRP6: low-density-lipoprotein receptor-related protein; Dvl: Dishevelled.

\section{Supplementary Material}

Supplementary figures and table. http://www.jcancer.org/v08p2523s1.pdf

\section{Acknowledgement}

This work was funded by Major science and technology projects of Zhejiang province (2014C03032), Key research project of traditional Chinese medicine science and technology plan in Zhejiang Province (2015ZZ007) and Wu Jieping foundation for clinical scientific research (320.6750.14300).

\section{Author contributions}

Jian $\mathrm{Hu}$ and Jinming $\mathrm{Xu}$ contributed to the conception and design of the work. Jinming $\mathrm{Xu}$ and Wang Lv contributed to conception, design, sample collection, data analysis and editing the manuscript. Yeji $\mathrm{Hu}$ and Luming Wang contributed to in vitro experiment, and interpretation of the data. Yiqing 
Wang and Jinlin Cao contributed to statistical analysis and revision of the manuscript. All authors have reviewed and approved the final draft of the manuscript.

\section{Competing Interests}

The authors have declared that no competing interest exists.

\section{References}

1. Torre LA, Bray F, Siegel RL, Ferlay J, Lortet-Tieulent J, Jemal A. Global cancer statistics, 2012. CA: a cancer journal for clinicians. 2015; 65: 87-108.

2. Chen W, Zheng R, Baade PD, Zhang S, Zeng H, Bray F, et al. Cancer statistics in China, 2015. CA: a cancer journal for clinicians. 2016; 66: 115-32.

3. Molina JR, Yang P, Cassivi SD, Schild SE, Adjei AA. Non-small cell lung cancer: epidemiology, risk factors, treatment, and survivorship. Mayo Clinic proceedings. 2008; 83: 584-94.

4. Torre LA, Siegel RL, Jemal A. Lung Cancer Statistics. Advances in experimental medicine and biology. 2016; 893: 1-19.

5. Perlikos F, Harrington KJ, Syrigos KN. Key molecular mechanisms in lung cancer invasion and metastasis: a comprehensive review. Critical reviews in oncology/hematology. 2013; 87: 1-11.

6. Gupta GP, Massague J. Cancer metastasis: building a framework. Cell. 2006; 127: 679-95.

7. Ocana OH, Corcoles R, Fabra A, Moreno-Bueno G, Acloque H, Vega S, et al. Metastatic colonization requires the repression of the epithelial-mesenchymal transition inducer Prrx1. Cancer cell. 2012; 22: 709-24.

8. Hugo H, Ackland ML, Blick T, Lawrence MG, Clements JA, Williams ED, et al. Epithelial-mesenchymal and mesenchymal-epithelial transitions in carcinoma progression. Journal of cellular physiology. 2007; 213: 374-83.

9. Singh A, Settleman J. EMT, cancer stem cells and drug resistance: an emerging axis of evil in the war on cancer. Oncogene. 2010; 29: 4741-51.

10. Fischer KR, Durrans A, Lee $\mathrm{S}$, Sheng J, Li F, Wong ST, et al. Epithelial-to-mesenchymal transition is not required for lung metastasis but contributes to chemoresistance. Nature. 2015; 527: 472-6.

11. Nakashima N, Liu D, Huang CL, Ueno M, Zhang X, Yokomise H. Wnt3 gene expression promotes tumor progression in non-small cell lung cancer. Lung cancer. 2012; 76: 228-34.

12. Li X, Xu Y, Chen $Y$, Chen S, Jia X, Sun $T$, et al. SOX2 promotes tumor metastasis by stimulating epithelial-to-mesenchymal transition via regulation of WNT / beta-catenin signal network. Cancer letters. 2013; 336: 379-89.

13. Gao Y, Song C, Hui L, Li CY, Wang J, Tian Y, et al. Overexpression of RNF146 in non-small cell lung cancer enhances proliferation and invasion of tumors through the Wnt/beta-catenin signaling pathway. PloS one. 2014; 9: e85377.

14. Xie C, Jiang G, Fan C, Zhang X, Zhang Y, Miao Y, et al. ARMC8alpha promotes proliferation and invasion of non-small cell lung cancer cells by activating the canonical Wnt signaling pathway. Tumour biology: the journal of the International Society for Oncodevelopmental Biology and Medicine. 2014; 35: 8903-11.

15. Li C, Song G, Zhang S, Wang E, Cui Z. Wnt3a increases the metastatic potential of non-small cell lung cancer cells in vitro in part via its upregulation of Notch3. Oncology reports. 2015; 33: 1207-14

16. Iasonos A, Schrag D, Raj GV, Panageas KS. How to build and interpret a nomogram for cancer prognosis. Journal of clinical oncology: official journal of the American Society of Clinical Oncology. 2008; 26: 1364-70.

17. Balachandran VP, Gonen M, Smith JJ, DeMatteo RP. Nomograms in oncology: more than meets the eye. The Lancet Oncology. 2015; 16: e173-80.

18. Camp RL, Dolled-Filhart M, Rimm DL. X-tile: a new bio-informatics tool for biomarker assessment and outcome-based cut-point optimization. Clinical cancer research: an official journal of the American Association for Cancer Research. 2004; 10: 7252-9.

19. Vickers AJ, Elkin EB. Decision curve analysis: a novel method for evaluating prediction models. Medical decision making: an international journal of the Society for Medical Decision Making. 2006; 26: 565-74.

20. Chaurasia A, Harel O. Using AIC in Multiple Linear Regression framework with Multiply Imputed Data. Health services \& outcomes research methodology. 2012; 12: 219-33.

21. Steyerberg EW, Vickers AJ. Decision curve analysis: a discussion. Medical decision making: an international journal of the Society for Medical Decision Making. 2008; 28: 146-9.

22. Li J, Liu $Y$, Yan Z, Wan X, Xia $Y$, Wang $K$, et al. A nomogram predicting pulmonary metastasis of hepatocellular carcinoma following partial hepatectomy. British journal of cancer. 2014; 110: 1110-7.

23. Steyerberg EW, Vergouwe Y. Towards better clinical prediction models: seven steps for development and an ABCD for validation. European heart journal. 2014; 35: 1925-31.

24. Talluri $\mathrm{R}$, Shete $\mathrm{S}$. Using the weighted area under the net benefit curve for decision curve analysis. BMC medical informatics and decision making. 2016; 16: 94 .
25. Tamai $K$, Zeng X, Liu C, Zhang X, Harada $Y$, Chang Z, et al. A mechanism for Wnt coreceptor activation. Molecular cell. 2004; 13: 149-56.

26. Zeng X, Tamai K, Doble B, Li S, Huang H, Habas R, et al. A dual-kinase mechanism for Wnt co-receptor phosphorylation and activation. Nature. 2005; 438: 873-7.

27. MacDonald BT, Tamai K, He X. Wnt/beta-catenin signaling: components, mechanisms, and diseases. Developmental cell. 2009; 17: 9-26.

28. Wang Z, Li B, Zhou L, Yu S, Su Z, Song J, et al. Prodigiosin inhibits Wnt/beta-catenin signaling and exerts anticancer activity in breast cancer cells. Proceedings of the National Academy of Sciences of the United States of America. 2016; 113: 13150-5.

29. Yang J, Weinberg RA. Epithelial-mesenchymal transition: at the crossroads of development and tumor metastasis. Developmental cell. 2008; 14: 818-29.

30. Lv YF, Dai H, Yan GN, Meng G, Zhang X, Guo QN. Downregulation of tumor suppressing STF cDNA 3 promotes epithelial-mesenchymal transition and tumor metastasis of osteosarcoma by the Wnt/GSK-3beta/beta-catenin/Snail signaling pathway. Cancer letters. 2016; 373: 164-73.

31. Hui L, Zhang S, Dong X, Tian D, Cui Z, Qiu X. Prognostic significance of twist and N-cadherin expression in NSCLC. PloS one. 2013; 8: e62171.

32. Zhao C, Li X, Su C, Li J, Cheng N, Ren S, et al. High expression of E-cadherin in pleural effusion cells predicts better prognosis in lung adenocarcinoma patients. International journal of clinical and experimental pathology. 2015; 8: 3104-9.

33. Zhong K, Chen W, Xiao N, Zhao J. The clinicopathological significance and potential drug target of E-cadherin in NSCLC. Tumour biology: the journal of the International Society for Oncodevelopmental Biology and Medicine. 2015; 36: 6139-48.

34. Yang YL, Chen MW, Xian L. Prognostic and clinicopathological significance of downregulated E-cadherin expression in patients with non-small cell lung cancer (NSCLC): a meta-analysis. PloS one. 2014; 9: e99763.

35. Coldren CD, Helfrich BA, Witta SE, Sugita M, Lapadat R, Zeng C, et al. Baseline gene expression predicts sensitivity to gefitinib in non-small cell lung cancer cell lines. Molecular cancer research: MCR. 2006; 4: 521-8.

36. Chung JH, Rho JK, Xu X, Lee JS, Yoon HI, Lee CT, et al. Clinical and molecular evidences of epithelial to mesenchymal transition in acquired resistance to EGFR-TKIs. Lung cancer. 2011; 73: 176-82.

37. Gupta PB, Onder TT, Jiang G, Tao K, Kuperwasser C, Weinberg RA, et al. Identification of selective inhibitors of cancer stem cells by high-throughput screening. Cell. 2009; 138: 645-59. 